\title{
Population Structure of the Fusarium graminearum Species Complex from a Single Japanese Wheat Field Sampled in Two Consecutive Years
}

G. W. Karugia, United Graduate School of Agricultural Sciences, Gifu University Yanagido 1-1, Gifu 501-1193, Japan; H. Suga, Life Science Research Centre, Gifu University, Yanagido 1-1, Gifu 501-1193, Japan; L. R. Gale, Department of Plant Pathology, University of Minnesota, St. Paul, MN 55108, USA; T. Nakajima, National Agricultural Research Center for Kyushu Okinawa Region, National Agriculture and Bio-oriented Research Organization, 2421 Suya, Kumamoto 861-1192 Japan; K. Tomimura, National Institute of Fruit Tree Science Kuchinotsu Region, 954 Kuchinotsu, Minamisimabara, Nagasaki 859-2501 Japan; and M. Hyakumachi, United Graduate School of Agricultural Sciences, Gifu University Yanagido 1-1, Gifu 501-1193, Japan

\begin{abstract}
Karugia, G. W., Suga, H., Gale, L. R., Nakajima, T., Tomimura, K., and Hyakumachi, M. 2009. Population structure of the Fusarium graminearum species complex from a single Japanese wheat field sampled in two consecutive years. Plant Dis. 93:170-174.

The Fusarium graminearum species complex ( $F g$ complex) that consists of at least 11 phylogenetically distinct species contains important Fusarium head blight (FHB) pathogens of wheat and barley worldwide. We obtained members of the $F g$ complex by sampling from a $500-\mathrm{m}^{2}$ experimental wheat field in Kumamoto Prefecture, Japan in two consecutive years and assessed them for species identity and trichothecene chemotype. Haplotype diversity was estimated by using 11 variable numbers of tandem repeat (VNTR) markers. In addition to these two samples (group 03W in 2003 and group 04W in 2004), pathogen populations from seed that was harvested in Fukuoka Prefecture and planted in the experimental field in 2002 (group 02WSC) and pathogen populations from seed that was harvested in Nagasaki Prefecture and planted in 2003 (group 03WSC) were analyzed for this study. Forty-six isolates were collected in each group. Most isolates from wheat heads were classified as $F$. asiaticum; only four isolates were classified as $F$. graminearum sensu stricto (s. str.). Out of a total of $183 \mathrm{Fg}$ complex strains, 80 isolates (44\%) were of the NIV type, while 103 isolates (56\%), including all four F. graminearum s. str. isolates, were of the 3ADON type. No 15ADON type isolate was detected in this study. Trichothecene chemotype compositions of $03 \mathrm{~W}$ and $04 \mathrm{~W}$ were nearly identical. High gene diversity of F. asiaticum was observed in all groups. Based on the observed low level of fixation index $\left(\mathrm{F}_{\mathrm{ST}}\right)$ and high level of effective number of migrants $(\mathrm{Nm})$, distinctive population subdivision of $F$. asiaticum was not inferred among the four groups. These results suggest that FHB in the experimental wheat field in both 2003 and 2004 was caused by a genetically similar population, which prevails in Kumamoto, Fukuoka, and Nagasaki prefectures.
\end{abstract}

Additional keywords: genetic comparison, Gibberella zeae, scab

In addition to causing quantitative yield losses, Fusarium head blight (FHB) of wheat and barley may result in grain contaminated with significant levels of trichothecene mycotoxins such as deoxynivalenol (DON) and the estrogenic mycotoxin, zearalenone. While FHB has been recognized as a worldwide threat to wheat and barley since the beginning of the twentieth century $(18,27)$, recent outbreaks have been reported in the United States, Canada, Europe, and Asia (11). Fusarium

Corresponding author: Haruhisa Suga E-mail: suga@gifu-u.ac.jp

* The $\boldsymbol{e}$-Xtra logo stands for "electronic extra" and indicates that a supplementary table is available online.

Accepted for publication 16 October 2008.

doi:10.1094/PDIS-93-2-0170

(C) 2009 The American Phytopathological Society graminearum, a species complex consisting of at least 11 phylogenetic species, some of which are dominant in particular geographical regions $(21,22,28)$, is the primary cause of FHB in many countries, including Japan (14,23), although many other Fusarium species and Microdochium nivale are also associated with FHB (15). Members of the $F$. graminearum species complex ( $F g$ complex) infect a broad range of other plant hosts in both temperate and subtropical areas (3). One species of the $F g$ complex, F. graminearum sensu stricto (s. str.), is cosmopolitan, while another species, $F$. asiaticum, is widespread in Asia $(8,21,22)$. In Japan, F. asiaticum and $F$. graminearum s. str. are the predominant species, although they are distributed unevenly geographically (30).

Members of the $F g$ complex are homothallic and can reproduce sexually, predominantly by selfing, but they can also out-cross (2). Genomic analyses based on sequencing suggest that out-crossing or hybridization between different species in the $F g$ complex occurs rarely in nature $(22,30)$, although $F$. asiaticum and $F$. graminearum s. str. have been successfully crossed in vitro (12). High genotypic diversity has been reported both for $F$. graminearum s. str. populations in the United States and F. asiaticum populations in eastern China, which may indicate that out-crossing within a species is frequent in nature $(8,25,35,36)$. High genotypic diversity of $F$. graminearum s. str. was even observed from small quadrants $\left(0.25 \mathrm{~m}^{2}\right)$ in a Kansas and a North Dakota wheat field based on amplified fragment length polymorphism (AFLP) marker data (35).

Individual isolates of the $F g$ complex produce one of three different trichothecene profiles, 3-acetyl deoxynivalenol (3ADON), 15-acetyl deoxynivalenol (15ADON), or nivalenol (NIV) (17). The different chemotypes may affect species or population ecology, as oxygenation or acetylation is known to alter the toxicity and bioactivity of trichothecenes $(1,13)$. Gale et al. (9) found population subdivision in $F$. graminearum s. str. in the United States that could be correlated with trichothecene chemotype.

Understanding the genetic profiles of pathogen populations may provide insights into the epidemiology and evolutionary potential of the $F g$ complex, and could lead to improved strategies for controlling this pathogen (35). Thus far, the population dynamics of $F g$ complex in a specific field is poorly understood.

The objective of this study was to assess the genetic diversity of species, trichothecene chemotypes, and VNTR haplotypes in $\mathrm{Fg}$ complex populations in seed lots in a $500-\mathrm{m}^{2}$ field in Kumamoto Prefecture, Japan and ensuing diseased wheat heads in two consecutive years.

\section{MATERIALS AND METHODS}

Isolates. A collection of 184 isolates of Fusarium spp. was established from seeds before planting and from symptomatic wheat heads after maturity at an experimental field $(10 \times 50 \mathrm{~m})$ of the National Agricultural Research Center for Kyushu Okinawa Region (KONRC; north latitude: 
32.879, east longitude: 130.742), Kumamoto Prefecture, Japan (Fig. 1; supplementary table). Wheat seeds of the variety Chikugo Izumi that were harvested from fields in Fukuoka Prefecture (Fig. 1) were planted in the experimental field in fall 2002 and matured in spring 2003. Wheat seeds harvested from fields in Nagasaki Prefecture (Fig. 1) were planted at the same experimental field in fall 2003 and matured in spring 2004. To isolate $F$. graminearum, symptomatic seed was sterilized by washing sequentially with $70 \%$ ethanol, $1 \%$ sodium hypochlorite, and sterile distilled water and placed on $F$. graminearum-selective medium modified from Komada's medium (32). Subsequent colonies were subcultured on synthetic nutrient agar (SNA). Conidia produced on SNA were spread on water agar, and a single conidium was isolated. Colonies showing typical morphologies for $F$. graminearum on potato sucrose agar (PSA), such as red pigmentation and fast growth, were used in this study. Isolates were maintained on potato dextrose agar (PDA) and kept at $6^{\circ} \mathrm{C}$ for short-term storage and at $-80^{\circ} \mathrm{C}$ in $50 \%$ glycerol for longterm storage. Forty-six isolates were obtained from each group in this study. The fungal populations isolated from seed planted in the experimental field in 2002 and 2003 were referred to as groups 02WSC and 03WSC, respectively. Populations from diseased wheat heads at the experimental field in 2003 and 2004 were referred to as groups $03 \mathrm{~W}$ and $04 \mathrm{~W}$, respectively. Diseased wheat heads in each of the 12 rows were collected at an interval of $10 \mathrm{~m}$ from the field edge (Fig. 1), and strains from wheat heads were named by location. For example, 03W5-10-1 referred to an isolate belonging to group $03 \mathrm{~W}$ from the fifth row of the site, $10 \mathrm{~m}$ away from the field edge and first in the site's wheat head serial numbers. Seeds were planted in lines without any specific spacing.

FHB in the experimental field was severe in 2003, and all 46 isolates were obtained from different sites in the field. In 2004 , one to four isolates were obtained from single sites in the field due to mild FHB occurrence. All isolates in both years were obtained from different wheat heads and about $10 \%$ of symptomatic seeds from each bulk harvest.

Genomic DNA extraction and PCRbased assessment of species and trichothecene type. Genomic DNA was extracted from 3- to 4-day-old mycelium (2 to $3 \mathrm{~cm}$ diameter) cultured in potato dextrose broth (PDB) as previously described (30). The final DNA pellet was dissolved in $200 \mu \mathrm{l}$ of water, and DNA yields were estimated based on comparison to DNA of known concentrations by agarose gel electrophoresis. All isolates were subjected to Fg complex-specific PCR with the UBC85F410 and UBC85R410 primer pair (24) and diagnostic PCR-restriction frag- ment length polymorphism (RFLP) for the distinction of $F$. asiaticum and $F$. graminearum s. str. with the primer pair H3dStyI and H3R1 and StyI or EcoRV as restriction enzyme (30). A multiplex PCR based on Tri12 sequences $(30,33)$ was performed to determine the trichothecene type of each isolate.

Generation of VNTR data. Eleven VNTR markers were used for isolate genotyping. Ten VNTR markers were developed previously (29). One additional VNTR marker, HK1003, developed by Gale et al. (7), was included. The forward primer for HK1003 is: 5'-ATCATATAG CACAGAACGGCG- ${ }^{\prime}$, the reverse primer is: 5'-TTACTCCAGCTTCACCGCAT-3'. The VNTR motif in the $F$. graminearum s. str. NRRL 31084 for this locus is nine repeats of GAATGGTAT followed by eight repeats of GAATGGTTC (Table 1). All forward primers were labeled with either fluorescent substance 6-FAM, VIC, NED, or PET to detect DNA fragments by ABI 3100 Genetic Analyzer (Applied Biosystems, Foster City, CA, USA) (Table 1). The total volume of the PCR reaction mixture was $10 \mu \mathrm{l}$, containing $10 \mathrm{mM}$ Tris- $\mathrm{HCl}$ at $\mathrm{pH} 8.3,50 \mathrm{mM} \mathrm{KCl}, 1.5 \mathrm{mM} \mathrm{MgCl}_{2}$,
$0.001 \%$ (wt $/ \mathrm{vol}$ ) gelatin, $200 \mu \mathrm{M}$ dNTP, $0.1-1.0 \mu \mathrm{M}$ of each of forward and reverse primer, 0.025 units Taq polymerase, and 1 $\mathrm{ng} / \mu \mathrm{l}$ genomic DNA. PCR was performed in an iCycler thermal cycler (Bio-Rad Laboratories, Hercules, CA, USA) using the following cycling parameters: $94^{\circ} \mathrm{C}$ for $2 \mathrm{~min}, 25$ cycles of $94^{\circ} \mathrm{C}$ for $1 \mathrm{~min}, 64^{\circ} \mathrm{C}$ for $1 \mathrm{~min}$, and $72^{\circ} \mathrm{C}$ for $1 \mathrm{~min}$. PCR products with different fluorescent labeled primers were mixed and diluted 20 -fold in water. Then, a 2- $\mu$ l sample was mixed with $8 \mu \mathrm{l}$ of HiDi Formamide including $0.25 \mu \mathrm{l}$ of 500 LIZ size standard (Applied Biosystems). Samples were loaded onto an ABI 3100 Genetic Analyzer with $50 \mathrm{~cm}$ capillary and POP 6 polymer (Applied Biosystems). The data were processed using the genotyping software Gene Mapper (Applied Biosystems). Allele types were represented according to detected fragment size compared to a $500 \mathrm{LIZ}$ standard.

Population genetic analyses. Most population genetic measures were computed using the software program Arlequin 2.000 (S. Schneider, D. Roessli, and L. Excoffier, Laboratoire de Génétique et Biometrie, Université de Genève, Switzer-

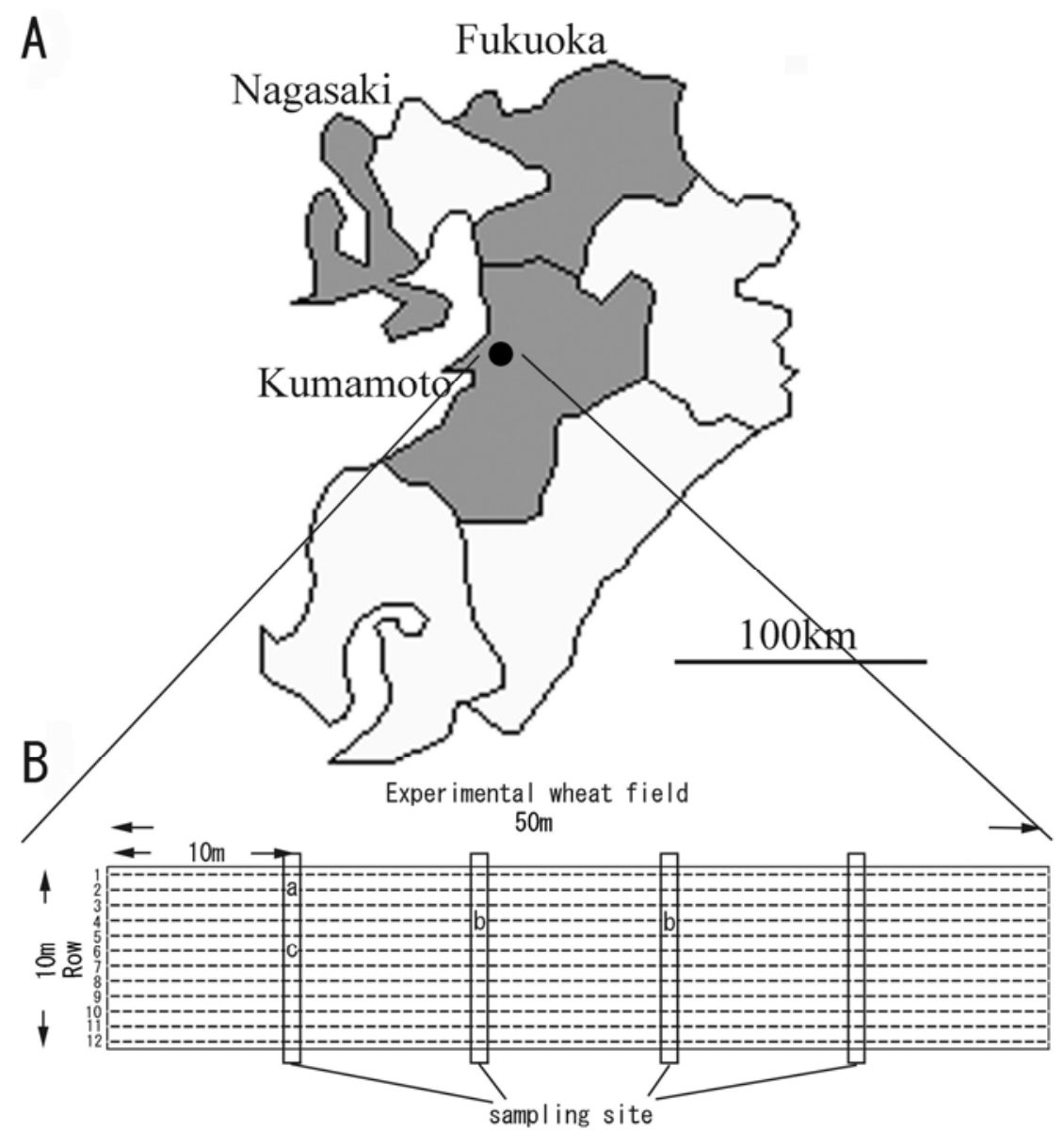

Fig. 1. A, Prefectures associated with collection of Fusarium graminearum species complex in Japan are shown in gray. The experimental field of the National Agricultural Research Center for Kyushu Okinawa Region (KONRC; north latitude: 32.879, east longitude: 130.742), Kumamoto Prefecture, is indicated by a circle. Seeds for planting in the experimental field were obtained from the Fukuoka and Nagasaki prefectures. B, Layout of experimental field. Isolates with same haplotype were obtained from 04W group at sites indicated by "a" (04W2-10-1 and 04W2-10-2), "b" (04W4-20-2 and 04W430-1), and "c" (04W6-10-1 and 04W6-10-3). 
land). Analysis of molecular variance (AMOVA) was applied to partition the genetic diversity into, among, and within population components (5). The fixation index $\left(\mathrm{F}_{\mathrm{ST}}\right)$ and effective number of migrants $(\mathrm{Nm})$ were used to infer genetic differentiation between populations. The $P$ value for linkage disequilibrium (26) that was developed from Fisher's exact probability test and implemented in Arlequin were obtained with the following conditions on the Markov chain length: 100,000; dememorization: 1,000 . The $P$ value for linkage disequilibrium was also computed using GenePop version 3.3 (M. Raymond and F. Rousset, Laboratoire de Génétique et Environnement, Université Montpellier, France) with the following Markov chain conditions: dememorization: 1,000 ; batches: 100; iterations per batch: 1,000. Nei's gene diversity (19) for each VNTR marker was computed using PopGene32 (34).

\section{RESULTS}

Species composition. Forty-six isolates were obtained for each group $(03 \mathrm{~W}, 04 \mathrm{~W}$, 02WSC, and 03WSC). Fg complex specific-PCR (24) was performed prior to the diagnostic PCR-RFLP test for $F$. graminearum s. str. and $F$. asiaticum (30). A DNA fragment of $332 \mathrm{bp}$ specific to the $F g$ complex (24) was amplified for all isolates except for DNA from isolate 04W10-10-3, which did not amplify. The diagnostic PCR-RFLP test indicated that most isolates (179 out of 184 ) were $F$. asiaticum (Table $2)$. Only four isolates, all from wheat heads, were $F$. graminearum s. str. (Table 2). Again, no amplification was observed for DNA of the 04W10-10-3 isolate. We concluded that this isolate may not be a member of $\mathrm{Fg}$ species complex and removed it from further analyses.

Trichothecene type composition. Out of 183 tested $\mathrm{Fg}$ complex isolates, 80 isolates $(44 \%)$ were of the NIV type as determined by the multiplex PCR $(30,33)$, while 103 isolates $(56 \%)$ and all four $F$. graminearum s. str. isolates were of the 3ADON type (Table 2). No 15ADON type isolate was detected in this study. In the head groups and in both years $(03 \mathrm{~W}$ and 04W), more NIV type isolates than $3 \mathrm{ADON}$ type were detected, in similar proportions in both years (Table 2).

Population analyses. Alleles for 11 VNTR markers were obtained for 183 isolates. All VNTR markers displayed polymorphisms among isolates (Table 1). The number of alleles (4 to 24) and the gene diversity in the whole sample (19) ( 0.15 to 0.94 ) were highly variable among

Table 1. Observed number of alleles and gene diversity in Japanese populations of the Fusarium graminearum species complex ${ }^{\text {a }}$

\begin{tabular}{lclcc}
\hline VNTR marker $^{\mathbf{b}}$ & Fluorescent label $^{\mathbf{c}}$ & Location $^{\mathbf{d}}$ & No of alleles $^{\text {Gene diversity }}$ \\
\hline HK1043 & 6-FAM & Ch1/Ct52/41,839 & $4^{\mathrm{f}}$ & $0.15(0.12)$ \\
HK913 & VIC & Ch1/Ct73/664 & $13^{\mathrm{f}}$ & $0.65(0.63)$ \\
HK917 & PET & Ch1/Ct82/2,471 & $16^{\mathrm{f}}$ & $0.81(0.81)$ \\
HK957 & NED & Ch1/Ct91/16,055 & 23 & $0.88(0.88)$ \\
HK1003 & PET & Ch1/Ct437/6,986 & 24 & $0.94(0.94)$ \\
HK965 & PET & Ch2/Ct154/51,671 & 15 & $0.83(0.82)$ \\
HK967 & VIC & Ch2/Ct154/53,868 & 5 & $0.28(0.27)$ \\
HK1059 & NED & Ch3/Ct196/164,228 & 6 & $0.66(0.66)$ \\
HK977 & NED & Ch3/Ct208/47,696 & $4^{\mathrm{f}}$ & $0.18(0.15)$ \\
HK630 & 6-FAM & Ch4/Ct371/112,325 & $6^{\mathrm{f}}$ & $0.33(0.30)$ \\
HK1073 & 6-FAM & Ch4/Ct398/70,812 & 8 & $0.53(0.52)$ \\
Mean & & & 10.36 & $0.57(0.55)$ \\
\hline
\end{tabular}

a Results based on analysis of 179 isolates of $F$. asiaticum and four isolates of $F$. graminearum s. str.

${ }^{\mathrm{b}}$ VNTR $=$ variable numbers of tandem repeat. HK1003 was initially described in Gale et al. (7), all others are from Suga et al. (29).

${ }^{c}$ Forward primer was fluorescent-labeled to obtain data by use of a 3100 Genetic Analyzer.

${ }^{\mathrm{d}}$ Marker location in the genome was indicated by Chromosome No./Contig No./Position in contig.

e Nei's gene diversity (h) (9) calculated in PopGene32 (34). Values in parentheses are for the 179 isolates of F. asiaticum only.

${ }^{\mathrm{f}}$ One allele was specific to isolates(s) of $F$. graminearum s. str. markers. HK1003 had the highest number of alleles and gene diversity among 11 VNTR markers (Table 1; supplementary table). Based on five VNTR markers, one of the alleles was specific to $F$. graminearum s. str. isolates. All but six out of 183 isolates represented unique VNTR haplotypes. The six isolates with shared haplotypes were all from the $04 \mathrm{~W}$ group. Two pairs of isolates (04W2-10-1 and 04W210-2, 04W6-10-1 and 04W6-10-3) were isolated from different heads at the same position in the field, but the pair 04W4-202 and 04W4-30-1 were isolated from field locations $10 \mathrm{~m}$ apart (Fig. 1). In addition to the wheat head populations $03 \mathrm{~W}$ and $04 \mathrm{~W}$, the seed populations 02WSC and 03WSC were included for population level analyses in order to compare the two genetic structures of these pathogen populations.

For further analyses, only VNTR data of 179 isolates of $F$. asiaticum were used since most isolates detected in this study were $F$. asiaticum, and limited gene flow between $F$. asiaticum and $F$. graminearum s. str. strains was presumed due to the presence of species-specific alleles in $F$. graminearum s. str. isolates (30). The average number of pairwise differences between isolates (5.717 to 6.275) and gene diversity ( 0.518 to 0.570 ) were similar for the four groups (Table 3). The majority $(99.28 \%)$ of the variance was caused by variation among isolates within populations. Low levels of pairwise $\mathrm{F}_{\mathrm{ST}}(0.002$ to 0.016 ) and a high level of effective number of migrants (Nm) (29.852 to 301.847) between groups were observed (Table 4). Based on a previous report (9) that population subdivision in $F$. graminearum s. str. in the midwestern United States was correlated with trichothecene type, we classified 179 F. asiaticum isolates into NIV $(\mathrm{n}=80)$ and $3 \mathrm{ADON}(\mathrm{n}=99)$ populations for additional analysis. $\mathrm{F}_{\mathrm{ST}}(0.060)$ and $\mathrm{Nm}$ (7.883) values suggested that population subdivision may be present between NIV and 3ADON trichothecene types, but it was not distinctive. Due to the lack of population subdivision, we considered all 179 isolates of $F$. asiaticum as part of a geographically larger population in this study, and the data were combined to assess the level of linkage disequilibrium between loci pairs. Significant linkage

Table 2. Species and trichothecene type composition for four Japanese populations of the Fusarium graminearum species complex

\begin{tabular}{|c|c|c|c|c|c|c|c|c|c|}
\hline \multirow[b]{3}{*}{ Group } & \multicolumn{9}{|c|}{ Species $^{\mathbf{a}}$} \\
\hline & \multicolumn{4}{|c|}{ Fusarium asiaticum } & \multicolumn{5}{|c|}{ Fusarium graminearum s. str. } \\
\hline & NIV $^{b}$ & 3ADON & 15ADON & Subtotal & NIV & 3ADON & 15ADON & Subtotal & Total \\
\hline 02WSC & 16 & 30 & 0 & 46 & 0 & 0 & 0 & 0 & 46 \\
\hline $03 \mathrm{~W}$ & 26 & 18 & 0 & 44 & 0 & 2 & 0 & 2 & 46 \\
\hline 03WSC & 13 & 33 & 0 & 46 & 0 & 0 & 0 & 0 & 46 \\
\hline $04 \mathrm{~W}$ & 25 & 18 & 0 & 43 & 0 & 2 & 0 & 2 & 45 \\
\hline Total & 80 & 99 & 0 & 179 & 0 & 4 & 0 & 4 & 183 \\
\hline
\end{tabular}

${ }^{a}$ Species determined by diagnostic polymerase chain reaction-restriction fragment length polymorphism (PCR-RFLP) for $F$. asiaticum and $F$. graminearum s. str. (30).

${ }^{\mathrm{b}}$ Trichothecene chemotype was determined by chemotype-specific multiplex PCR $(30,33)$. Number of isolates is indicated. 
disequilibrium $(P<0.05)$ was detected in $10(18 \%)$ and $12(22 \%)$ out of 55 combinations by Arlequin ver. 2.000 and GenePop ver. 3.3 , respectively (Table 5).

\section{DISCUSSION}

Although population genetic studies of the $\mathrm{Fg}$ complex have previously been reported in the United States and eastern China $(8,9,25,35,36)$, population dynamics of this fungal complex remains poorly understood in other countries. We assessed genetic profiles of the $\mathrm{Fg}$ complex from infested seed lots and from a wheat field in Kumamoto, Japan in two consecutive years. Efficacy and adequacy of 11 VNTR markers $(7,29)$ for population level analyses of the $F g$ complex were also confirmed in this study.

Sample collection sites for the four groups were all located in the Kyushu area (Fig. 1). Previous studies of the $\mathrm{Fg}$ complex on wheat or barley revealed the presence and differential geographical distribution of $F$. asiaticum and $F$. graminearum s. str. in Japan $(21,30)$. The prevalence of $F$. asiaticum in all samples in this study confirmed the dominance of $F$. asiaticum in southern Japan, including Kyushu (30). Although the previous study did not detect any $F$. graminearum s. str. isolates from Kyushu, F. graminearum s. str. was detected at low frequency in this study. The co-existence of both $F$. asiaticum and $F$. graminearum s. str. provides a potential for introgression (hybridization) in the field. Progeny from crosses between these two species have been obtained previously in vitro (12). However, the presence of alleles specific to isolates of $F$. graminearum s. str. (Table 1) suggests these two species live sympatrically, supporting results from a previous study in Tohoku, Japan where both species co-occur. Similarly, no apparent hybrids were detected in that study (30). Nevertheless, we currently cannot rule out occasional or low levels of hybridization between these species.

NIV type strains of $F$. graminearum s. str. have not been detected in Japan (30), but global surveys previously identified all three trichothecene types (NIV, 3ADON, 15ADON) in both $F$. asiaticum and $F$. graminearum s. str. $(30,33)$. In this study, $F$. asiaticum isolates were either NIV or $3 \mathrm{ADON}$ types, and all four $F$. graminearum s. str. isolates were the $3 \mathrm{ADON}$ type (Table 2). Population subdivisions correlated with $15 \mathrm{ADON}$ and $3 \mathrm{ADON}$ trichothecene types in $F$. graminearum s. str. were detected in the midwestern United States (9). We hypothesized that Japanese population subdivisions of $F$. asiaticum may be correlated with NIV or 3ADON. Although no clear correlation between haplotypes and chemotypes was found, organization of isolates based on trichothecene types nevertheless resulted in higher levels of population subdivision $\left(\mathrm{F}_{\mathrm{ST}}=0.060\right)$ and in lower number of migrants $(\mathrm{Nm}=7.883)$ compared to other groupings. More intensive sampling from a wider area is probably needed for further insight.

Most studies have demonstrated high genotypic diversity in collections of $F$.

Table 3. Mean number of pairwise differences and gene diversity $(\mathrm{H})$ across 11 variable numbers of tandem repeat (VNTR) markers in four Japanese populations of Fusarium asiaticum

\begin{tabular}{lccc}
\hline Group $^{\mathbf{a}}$ & No. of isolates $^{\mathbf{b}}$ & $\begin{array}{c}\text { Pairwise differences } \\
\text { and SD }^{\mathbf{c}}\end{array}$ & H and SD $^{\mathbf{d}}$ \\
\hline 02WSC & 46 & $5.717(2.789)$ & $0.518(0.281)$ \\
03W & 44 & $6.275(3.035)$ & $0.570(0.306)$ \\
03WSC & 46 & $6.169(2.987)$ & $0.561(0.301)$ \\
04W & 43 & $6.213(3.010)$ & $0.565(0.304)$ \\
Total & 179 & $6.123(2.923)$ & $0.577(0.294)$ \\
\hline
\end{tabular}

a 02WSC were from seeds harvested at Fukuoka Prefecture and planted in the experimental fields in Kumamoto Prefecture in 2002. 03WSC isolates were from seeds harvested at Nagasaki Prefecture and planted in 2003. $03 \mathrm{~W}$ and $04 \mathrm{~W}$ isolates were from diseased wheat heads in the experimental field in 2003 and 2004, respectively.

${ }^{\mathrm{b}} F$. asiaticum isolates.

${ }^{\mathrm{c}}$ Mean number of pairwise differences and standard deviation (SD) calculated using Arlequin version 2.000 (S. Schneider, D. Roessli, and L. Excoffier, Laboratoire de Génétique et Biometrie, Université de Genève, Switzerland).

${ }^{d}$ Average gene diversity over loci and SD (in parentheses) was determined according to Nei (20) and Tajima (31).

Table 4. Pairwise $\mathrm{F}_{\mathrm{ST}^{\mathrm{a}}}{ }^{\mathrm{a}}$ (above diagonal) and effective number of migrants $(\mathrm{Nm})^{\mathrm{b}}$ (below diagonal) between populations of Fusarium asiaticum

\begin{tabular}{lcccc}
\hline & 02WSC & 03W & 03WSC & 04W \\
\hline 02WSC & $\ldots$ & 0.009 & 0.003 & 0.016 \\
03W & 57.230 & $\ldots$ & 0.002 & 0.005 \\
03WSC & 198.838 & 301.847 & $\ldots$ & 0.009 \\
04W & 29.852 & 100.503 & 53.025 & $\ldots$ \\
\hline
\end{tabular}

${ }^{\mathrm{a}} \mathrm{F}_{\mathrm{ST}}$ values were calculated using Arlequin version 2.000 (S. Schneider, D. Roessli, and L. Excoffier, Laboratoire de Génétique et Biometrie, Université de Genève, Switzerland).

${ }^{\mathrm{b}}$ Effective number of migrants (Nm) was calculated using Arlequin version 2.000 that implements a concept developed by Nei (20). graminearum (8). Zeller et al. (35) analyzed $F$. graminearum from diseased wheat heads in small $\left(0.25 \mathrm{~m}^{2}\right)$ quadrants in Kansas and North Dakota by AFLP. Among 253 tested isolates, 169 were of a unique haplotype. High genotypic diversity of $F$. asiaticum was also observed in this study. VNTR data indicated that 88 out of 91 isolates (including the four $F$. graminearum s. str. isolates) from the $500-\mathrm{m}^{2}$ wheat field $(03 \mathrm{~W}$ and $04 \mathrm{~W})$ and all 92 isolates from seeds (02WSC and 03WSC) were of a unique haplotype. Identical haplotypes, which were apparently clonal, have been recovered from neighboring plants with low frequency $(8,35)$. Three pairs of isolates that shared VNTR haplotypes were detected in the $04 \mathrm{~W}$ sample but not in the 03W sample. Different sampling strategies used in $03 \mathrm{~W}$ and $04 \mathrm{~W}$ may have been influential. For $03 \mathrm{~W}$, all isolates were obtained from different sites in the field, but due to the mild FHB occurrence in 2004, one to four isolates were obtained from single sites in the $04 \mathrm{~W}$ sample. However, our results did not contradict the hypothesis that most infections are initiated by genotypically diverse spores, followed by limited secondary infection $(8,35)$. Occurrence of the same haplotypes in different heads can be explained by secondary spread of asexual conidia, a local primary inoculum source, or asexual conidia and/or ascospores derived from selfing.

Of 55 paired combinations between the 11 VNTR markers, $10(18 \%)$ or $12(22 \%)$ marker pairs were in linkage disequilibrium. Of 78 possible combinations between 13 AFLP loci, four locus pairs (5\%) in the Kansas and eight locus pairs (10\%) in the North Dakota population were in

Table 5. Observed significant linkage disequilibrium $(P<0.05)$ between 11 variable numbers of tandem repeat (VNTR) markers in 179 isolates of Fusarium asiaticum

\begin{tabular}{lcc}
\hline & \multicolumn{2}{c}{$\boldsymbol{P}$ value } \\
\cline { 2 - 3 } Marker pair & Arlequin $^{\mathbf{a}}$ & GenePop $^{\mathbf{b}}$ \\
\hline HK913, HK917 & 0.000 & 0.000 \\
HK913, HK957 & 0.000 & 0.000 \\
HK913, HK965 & 0.001 & 0.000 \\
HK917, HK1003 & 0.009 & 0.047 \\
HK917, HK1059 & 0.007 & 0.010 \\
HK957, HK965 & 0.000 & 0.002 \\
HK1003, HK630 & 0.032 & 0.029 \\
HK967, HK1059 & 0.022 & 0.011 \\
HK977, HK630 & 0.014 & 0.016 \\
HK1059, HK1073 & 0.001 & 0.001 \\
HK967, HK1073 & $0.056^{c}$ & 0.048 \\
HK1043, HK917 & $0.078^{\text {c }}$ & 0.047 \\
\hline
\end{tabular}

${ }^{\mathrm{a}}$ In Arlequin version 2.000 (S. Schneider, D. Roessli, and L. Excoffier, Laboratoire de Génétique et Biometrie, Université de Genève, Switzerland), a test by Slatkin (26) based on Fisher's exact probability test is implemented.

${ }^{\mathrm{b}}$ In GenePop version 3.3 (M. Raymond and F. Rousset, Laboratoire de Génétique et Environnement, Université Montpellier, France) Fisher's exact probability test is implemented.

${ }^{c}$ Not significant by Arlequin version 2.000 but significant by GenePop version 3.3. 
linkage disequilibrium in a previous study (35). Low levels of linkage disequilibrium may be interpreted as evidence for frequent out-crossing. However, it might also be attained by maintenance of high genetic diversity in large populations. Gale et al. (8) found linkage disequilibrium $(P<0.05)$ on 13 locus pairs $(36 \%)$ of 36 possible combinations between six RFLP loci in an eastern China population. Marker position is a conceivable cause of linkage disequilibrium, although markers HK965 and HK967 are only separated from each other by 2,200 bp according to the genome database of $F$. graminearum s. str. (Table 1). Surprisingly, significant linkage disequilibrium between these two markers was not found in this study $(P=0.425$ in Arlequin, $P=0.329$ in GenePop). In the 2.2-kb $\alpha_{1}$-antitrypsin locus in humans, linkage disequilibrium was low between alleles at three RFLPs (4). Alternatively, the genome construction of marker regions in $F$. asiaticum may be different from that of $F$. graminearum s. str.

Actual frequencies of intraspecies outcrossing in nature remain unclear. Genetic profile analyses of ascospores derived from perithecia collected from fields could give more direct evidence of the prevalence of intraspecies out-crossing.

FHB may be initiated by regional inoculum. However, the contribution of spores transported long-distance to FHB epidemics has also been discussed $(6,16,25)$. Gilbert et al. (10) showed that Fusarium-infested seed was not a major cause of FHB in the crop year it was planted. Schmale et al. (25) suggested that FHB inoculum may originate from multiple locations over large geographic areas. For F. asiaticum, the contribution of the seed population to FHB in this study could not be attained because distinctive population subdivisions between the groups were not detected (Table 4). However, the lack of distinctive population subdivisions among the four groups at least indicated that FHB in both 2003 and 2004 was caused by a genetically homogeneous population that prevails in Kumamoto, $\mathrm{Fu}-$ kuoka, and Nagasaki prefectures.

\section{ACKNOWLEDGMENTS}

We thank Joseph Mwafaida of Pwani University, Kilifi, Kenya for critically reading the manuscript. This work was supported in part by a Grant-in-Aid for Scientific Research from the Ministry of Education, Culture, Sports, Science, and Technology of Japan (No. 16780224) and was also conducted as part of the project, Development and Evaluation of Management Methods for Supplying Safe, Reliable and Functional Food and Farm Produce, funded by the National Food Research Institute.

\section{LITERATURE CITED}

1. Alexander, N. J., Hohn, T. M., and McCormick, S. P. 1998. The TRI11 gene of Fusarium sporotrichioides encodes a cytochrome P-450 monooxygenase required for $\mathrm{C}-15$ hydroxylation in trichothecene biosynthesis. Appl. Environ. Microbiol. 64:221-225.

2. Bowden, R. L., and Leslie, J. F. 1999. Sexual recombination in Gibberella zeae. Phytopathology 89:182-188.

3. Cook, R. J. 1981. Fusarium diseases of wheat and other small grains in North America. Pages 39-52 in: Fusarium: Diseases, Biology and Taxonomy. P. E. Nelson, T. A. Toussoun and R. J. Cook, eds. Pennsylvania State University Press, University Park.

4. Cox, D. W., Woo, S. L. C., and Mansfield, T. 1985. DNA restriction fragments associated with $\alpha_{1}$-antitrypsin indicate a single origin for deficiency allele $P I Z$. Nature 316:79-81.

5. Excoffier, L., Smouse, P., and Quattro, J. 1992. Analysis of molecular variance inferred from metric distances among DNA haplotypes, application to human mitochondrial DNA restriction data. Genetics 131:479-491.

6. Francl, L., Shaner, G., Bergstrom, G., Gilbert, J., Pedersen, W., Dill-Macky, R., Sweets, L., Corwin, B., Jin, Y., Gallenberg, D., and Wiersma, J. 1999. Daily inoculum levels of Gibberella zeae on wheat spikes. Plant Dis. 83:662-666.

7. Gale, L. R., Bryant, J. D., Calvo, S., Giese, H., Katan, T., O'Donnell, K., Suga, H., Taga, M., Usgaard, T. R., Ward, T. J., and Kistler, H. C. 2005. Chromosome complement of the fungal plant pathogen Fusarium graminearum based on genetic and physical mapping and cytological observations. Genetics 171:985-1001.

8. Gale, L. R., Chen, L.-F., Hernick, C. A., Takamura, K., and Kistler, H. C. 2002. Population analysis of Fusarium graminearum from wheat fields in eastern China. Phytopathology 92:1315-1322.

9. Gale, L. R., Ward, T. J., Balmas, V., and Kistler, H. C. 2007. Population subdivision of Fusarium graminearum sensu stricto in the upper Midwestern United States. Phytopathology 97:1434-1439.

10. Gilbert, J., Conner, R. L., Fernandez, M. R., McLaren, D., and Woods, S. M. 2003. Role of spring wheat seed infested with Fusarium graminearum in spread and development of fusarium head blight and effects on agronomic performance. Plant Pathol. 25:73-81.

11. Goswami, R. S., and Kistler H. C. 2004. Heading for disaster: Fusarium graminearum on cereal crops. Mol. Plant Pathol. 5:515-525.

12. Jurgenson, J. E., Bowden, R. L., Zeller, K. A., Leslie, J. F., Alexander, N. J., and Plattner, R. D. 2002. A genetic map of Gibberella zeae (Fusarium graminearum). Genetics 160:14511460.

13. Kimura, M., Kaneko, I., Komiyama, M., Takatsuki, A., Koshino, H., Yoneyama, K., and Yamaguchi, I. 1998. Trichothecene 3-Oacetyltransferase protects both the producing organism and transformed yeast from related mycotoxins. J. Biol. Chem. 273:1654-1661.

14. Koizumi, S., Kato, H., Yoshino, R., Komada, H., Ichinoe, M., Umehara, Y., and Hayashi, N. 1993. Studies on the pathogens and epidemiology of wheat and barley scab. Bull. Natl. Agric. Res. Center Kyushu Okinawa Region (KONRC) 23:1-104.

15. Liddell, C. M. 2003. Systematics of Fusarium species and allies associated with Fusarium head blight. Pages 35-43 in: Fusarium Head Blight of Wheat and Barley. K. J. Leonard and W. R. Bushnell, eds. American Phytopathological Society, St. Paul, MN.

16. Maldonado-Ramirez, S. L., Schmale, D. G., Shields, E. J., and Bergstrom, G. C. 2005. The relative abundance of viable spores of Gibberella zeae in the planetary boundary layer suggests the role of long-distance transport in regional epidemics of Fusarium head blight. Agric. For. Meteorol. 132:20-27.

17. Miller, J. D., Greenhalgh, R., Wang, Y., and Lu, M. 1991. Trichothecene chemotypes of three Fusarium species. Mycologia 83:121-130.

18. Muriuki, J. G. 2001. Deoxynivalenol and nivalenol in pathogenesis of Fusarium head blight in wheat. Ph.D. thesis. University of Minnesota, St. Paul.

19. Nei, M. 1973. Analysis of gene diversity in subdivided populations. Proc. Natl. Acad. Sci. USA 70:3321-3323.

20. Nei, M. 1987. Molecular evolutionary genetics. Columbia University Press, New York.

21. O'Donnell, K., Kistler, H. C., Tacke, B. K., and Casper, H. H. 2000. Gene genealogies reveal global phylogeographic structure and reproductive isolation among lineages of Fusarium graminearum, the fungus causing wheat scab. Proc. Natl. Acad. Sci. USA 97:7905-7910.

22. O'Donnell, K., Ward, T. J., Geiser, D. M., Kistler, H. C., and Aoki, T. 2004. Genealogical concordance between the mating type locus and seven other nuclear genes supports formal recognition of nine phylogenetically distinct species within the Fusarium graminearum clade. Fungal. Genet. Biol. 41:600-623.

23. Parry, D. W., Jenkinson, P., and MacLeod, L. 1995. Fusarium ear blight (scab) in small grain cereals-a review. Plant Pathol. 44:207-238.

24. Schilling, A. G., Moller, E. M., and Geiger, H. H. 1996. Polymerase chain reaction-based assays for species-specific detection of Fusarium culmorum, $F$. graminearum, and $F$. avenaceum Phytopathology 86:515-522.

25. Schmale, D. G., III., Leslie, J. F., Zeller, K. A., Saleh, A. A., Shields, E. J., and Bergstrom, G. C. 2006. Genetic structure of atmospheric populations of Gibberella zeae. Phytopathology 96:1021-1026.

26. Slatkin, M. 1994. Linkage disequilibrium in growing and stable populations. Genetics 137:331-336.

27. Stack, R. W. 2003. History of Fusarium head blight with emphasis on North America. Pages 134 in: Fusarium Head Blight of Wheat and Barley. K. J. Leonard and W. R. Bushnell, eds. American Phytopathological Society, St. Paul, MN.

28. Starkey, D. E., Ward, T. J., Aoki, T., Gale, L. R., Kistler, H. C., Geiser, D. M., Suga, H., Tóth, B., Varga, J., and O'Donnell, K. 2007. Global molecular surveillance reveals novel Fusarium head blight species and trichothecene toxin diversity. Fungal. Genet. Biol. 1191-1204.

29. Suga, H., Gale, L. R., and Kistler, H. C. 2004. Development of VNTR markers for two Fusarium graminearum clade species. Mol. Ecol. Notes 4:468-470

30. Suga, H., Karugia, G. W., Ward, T., Gale, L. R., Tomimura, K., Nakajima, T., Miyasaka, A., Koizumi, S., Kageyama, K., and Hyakumachi, M. 2008. Molecular characterization of the Fusarium graminearum species complex in Japan. Phytopathology 98:159-166.

31. Tajima, F. 1993. Measurement of DNA polymorphism. Pages 37-59 in: Mechanisms of Molecular Evolution. Introduction to Molecular Paleo-population Biology. N. Tanaka and A. G. Clark, eds. Sinauer Associates, Sunderland, MA

32. Togawa, M. 1994. Selective medium for isolation of Fusarium graminearum. Soil Microorg. 44:77-88.

33. Ward, T. J., Bielawski, J. P., Kistler, H. C., Sullivan, E., and O'Donnell, K. 2002. Ancestral polymorphism and adaptive evolution in the trichothecene mycotoxin gene cluster of phytopathogenic Fusarium. Proc. Natl. Acad. Sci. USA 99:9278-9283.

34. Yeh, F. C., Yang, R., Boyle, T. J., Ye, Z., and Xiyan, J. M. 2000. PopGene32, Microsoft Windows-based freeware for population genetic analysis, version 1.32. Molecular Biology and Biotechnology Centre, University of Alberta, Edmonton, Alberta, Canada.

35. Zeller, K. A., Bowden, R. L., and Leslie, J. F. 2003. Diversity of epidemic populations of Gibberella zeae from small quadrants in Kansas and North Dakota. Phytopathology 93:874-880.

36. Zeller, K. A., Bowden, R. L., and Leslie, J. F. 2004. Population differentiation and recombination in wheat scab populations of Gibberella zeae from the United States. Mol. Ecol. 13:563-571. 